\title{
Compensated magnetism by design in double perovskite oxides
}

\author{
Victor Pardo ${ }^{1,2, *}$ and Warren E. Pickett ${ }^{1}$ \\ ${ }^{1}$ Department of Physics, University of California, Davis, CA 95616 \\ ${ }^{2}$ Departamento de Física Aplicada, Facultad de Física, Universidad de Santiago de Compostela, \\ E-15782 Campus Sur s/n, Santiago de Compostela, Spain
}

(Dated: October 30, 2018)

\begin{abstract}
Taking into account Goodenough's superexchange rules, including both full structural relaxation and spin-orbit coupling, and checking strong correlation effects, we look for compensated half metals within the class of oxide double perovskites materials. Identifying likely half metallic (or half semimetallic) antiferromagnets, the full complications including orbital magnetism are included in order to arrive at realistic predictions of designed magnetic compounds with (near) vanishing net moment. After sorting through several candidates that have not been considered previously, two materials, $\mathrm{K}_{2} \mathrm{MnRhO}_{6}$ and $\mathrm{La}_{2} \mathrm{CrWO}_{6}$, remain as viable candidates. An important factor is obtaining compounds either with very small induced orbital moment (helped by closed subshells) or with an orbital moment that compensates the spin-orbit driven degradation of half metallic character. While thermodynamic stability of these materials cannot be ensured, the development of layer-by-layer oxide deposition techniques does not require that materials be thermodynamically stable to be synthesized.
\end{abstract}

PACS numbers:

\section{INTRODUCTION}

Double perovskites have drawn considerable attention in the field of spintronics, particularly since the discovery $\underline{\underline{1}}$ of colossal magnetoresistance in $\mathrm{Sr}_{2} \mathrm{FeMoO}_{6}$ that is believed to be a half metallic (HM) ferromagnet (FM). The HM property is highly desired in spintronics applications, where spin currents are utilized as well as charge currents. The double perovskite family of oxides has been one of the most popular classes within which to look for HMFMs $\stackrel{2}{2}$

Recently, with wider application of deposition techniques leading to better materials and improved understanding, layer by layer deposition of perovskite oxides has led to unusual and potentially useful physical properties $\frac{3}{3}$ Most such depositions of perovskites have used the (001) growth direction, resulting in a wide variety of oxide heterostructures with tetragonal symmetry. Double perovskite materials can be considered as single unit cell, multilayered perovskite structures that have been grown along the (111) direction, and such growth is a promising direction for synthesizing new members of this class.

Although HMFM character will provide new (and highly sought after) magnetoelectronic properties, there is a subclass that is more exotic. When half metallicity occurs and in addition the moments compensate, a "compensated half metal" (historically called a half metallic antiferromagnet ${ }^{4}$ ) arises. This state has magnetic order but vanishing macroscopic spin magnetization, it could support a new type of superconductivity, $\stackrel{5}{5}$ and one can imagine numerous possible applications for a half metal that is relatively impervious to external magnetic fields. However, this spin-only picture is an idealization; spinorbit coupling (SOC) couples the two spin directions and thereby destroys the precise spin compensation, and in addition generates spin-induced orbital moments. Thus a fully compensated half metal with vanishing macroscopic magnetization is an idealization, and the focus must be on finding realizations that are as close as possible to full compensation.

Interest has been rekindled recently in the effects of spin-orbit coupling in oxides because, unlike the degradation of half metallicity that is commonly discussed and expected, it can also lead to more exotic effects in the spin- and orbital-magnetism, especially in $d^{1}$ or $\mathrm{d}^{5}$ ions in $t_{2 g}$ subshells where large orbital moments may arise $\underline{6}$ A dramatic possibility is the promotion of compensating spin and orbital moments. In $\mathrm{Ba}_{2} \mathrm{NaOsO}_{6}$, for example, calculations ${ }^{7}$ indicate that the $d^{1}$ spin moment of Os is compensated by the $t_{2 g} \mathrm{~L}=1$ orbital moment induced by $\mathrm{SOC}$, and it is the partial orbital quenching by the environment that destroys the compensation of the moment. In $\mathrm{Sr}_{2} \mathrm{CrOsO}_{6}$, neglect of $\mathrm{SOC}$ leads to a semimetallic compensated half metal, which is destroyed by the large SOC in this system. ${ }^{8}$ In isovalent $\mathrm{Sr}_{2} \mathrm{CrRuO}_{6}$, where weaker SOC arises on $\mathrm{Ru}$ compared with Os, the reduced spin and induced orbital moments conspire to nearly cancel, giving a real possibility for an effectively compensated half metal. In correlated insulators, strong SOC can lead to much more complex magnetic coupling, thereby complicating the resulting magnetic order ${ }^{9}$ and reducing the likelihood of achieving a compensated 
half metal.

Half-metallic antiferromagnetic (HMAF) materials have not been obtained experimentally yet. Several theoretical efforts have been carried out in predicting half-metallic antiferromagnetism, but experimentally, this state remains elusive. HMAFs have been predicted for various double perovskites, $10,11,12,13$ tetrahedrally coordinated compounds 14 and for Heusler-structured materials, 15,16,17 Design of materials with desired properties is a long-standing hope that is gaining momentum,, 18 and the considerable experience that has been accumulated in oxide double perovskites provides guidelines to focus design efforts.

In this paper we build on previous studies, and extend the earlier work by considering all of factors: ion size, structural relaxation, and SOC. Our general strategy is:

i) we focus on the double perovskite structure, with the chemical formula $\mathrm{A}_{2} \mathrm{MM}^{\prime} \mathrm{O}_{6}$, where $\mathrm{M}$ and $\mathrm{M}$ ' are transition metal ions lying on a rocksalt type sublattice. An ordered double perovskite is more likely to occur if $\mathrm{M}$ and $\mathrm{M}$ ' differ in ionic radius, and differ in formal charge.

ii) we use ab initio calculations to relax the structure, including volume optimization, $c / a$ optimization, and internal coordinate relaxation, thereby obtaining structures that are dynamically stable, and energetically metastable if not stable.

iii) we include SOC, which mixes the spin moment with an orbital moment, and also analyze the effects of correlation corrections by using the LSDA+U method, $, 19,20$ to check the dependence of the magnetic and electronic structure properties on $U$ (the on-site Coulomb repulsion). The objective is to determine to what degree the spin-compensation is maintained, and to understand the interplay of structural changes, SOC, and effects of electronic correlation.

We use the Goodenough-Kanamori-Anderson (GKA) rules for superexchange ${ }^{21}$ for the $180^{\circ}$ metaloxygen-metal bond occurring in perovskites to guide our choices. According to these authors, and the expected moments, only a few $\mathrm{d}^{n}-\mathrm{d}^{m}$ electronic configurations that would lead to a spin-compensated situation are likely. In addition, we aim to maximize the likelihood of obtaining an ordered perovskite by mixing a $3 \mathrm{~d}$ row element with another metal cation from the $4 \mathrm{~d}$ or $5 \mathrm{~d}$ row, so that the size mismatch will make chemical ordering more likely. According to GKA, the possible antiferromagnetically coupled electronic configurations are: $\mathrm{d}^{2}-\mathrm{d}^{2}, \mathrm{~d}^{2}-\mathrm{d}^{4}(\mathrm{LS}), \mathrm{d}^{3}-$ $\mathrm{d}^{3}, \mathrm{~d}^{8}-\mathrm{d}^{8}$ and other combinations that include in all cases high spin (HS) cations: $\mathrm{d}^{4}-\mathrm{d}^{4}, \mathrm{~d}^{5}-\mathrm{d}^{5}, \mathrm{~d}^{4}-\mathrm{d}^{6}$, $d^{6}-d^{6}, d^{7}-d^{7}$. These latter options have not been pursued because it is difficult to stabilize HS states with those large moments for the $4 \mathrm{~d}$ and $5 \mathrm{~d}$ elements, which usually take on a higher valence state than the $3 \mathrm{~d}$ element, and hence the relatively strong crystal field will favor low-spin states. Particularly interesting is the $\mathrm{d}^{3}-\mathrm{d}^{3}$ configuration when SOC is taken into account. Since it deals with a completely filled $t_{2 g}$ band, the effective angular momentum will be very small, although there is also the possibility of insulating states. We will discuss this issue later in the paper.

\section{COMPUTATIONAL DETAILS}

Electronic structure calculations were performed within density functional theory ${ }^{22}$ using WIEN $2 \mathrm{~K}, \stackrel{23}{,}$ which utilizes an augmented plane wave plus local orbitals $(\mathrm{APW}+\mathrm{lo})^{24}$ method to solve the KohnSham equations. This method uses an all-electron, full-potential scheme that makes no shape approximation to the potential or the electron density. The exchange-correlation potential used was the Perdew and Wang version of the local density approximation 25 and strong correlation effects were introduced by means of the LSDA+U scheme 26,27 including an on-site $\mathrm{U}$ (Coulomb repulsion) and $\mathrm{J}$ (Hund's rule exchange) for the metal d states. We have used option nldau= 1 in WIEN2K, i.e. the socalled "fully-localized limit". Structural optimizations (unless otherwise stated) were performed using the Perdew-Burke-Ernzerhof version of the generalized gradient approximation ${ }^{28}$ Spin-orbit coupling was introduced using the scalar relativistic approximation. All the calculations were converged with respect to all the parameters used, to the precision necessary to support our calculations (converged forces and total energy differences), up to $\mathrm{R}_{m t} \mathrm{~K}_{\max }=7$ and an $8 \times 8 \times 8 \mathrm{k}$-mesh.

\section{REVISITING PREVIOUSLY FOUND MATERIALS}

\section{A. $\mathrm{La}_{2} \mathrm{VCuO}_{6}: \mathbf{d}^{1}-\mathbf{d}^{9}$}

It had been reported previously that this material is a HMAF, 10,29 but previous studies did not consider the structural relaxation that we have now carried out, and that is expected to be important in this strongly Jahn-Teller active system. At the LSDA+U level, this compound converges to a HMAF, which remains when one relaxes the structure. We have done a relaxation within a tetragonal symmetry (but the ratio $c / a \sim 1.00$, to $1 \%$ accuracy, as expected), obtaining $a=3.64 \AA$. The internal structure relaxes to accommodate the Jahn-Teller distortion of the 
$\mathrm{Cu}^{2+}$ and $\mathrm{V}^{4+}$ cations. Depending on the value of $\mathrm{U}$ chosen (the same $\mathrm{U}$ was chosen for both cations in our calculations), this distortion varies, being larger for a larger U. For relatively small values of $U$ (effective $\mathrm{U}$ of only $4 \mathrm{eV}$ ) there is a large Jahn-Teller distortion, the oxygen octahedra around the $\mathrm{Cu}^{2+}$ cation become elongated by about $4 \%$ to accommodate the $\mathrm{d}^{9}$ cation and around $\mathrm{V}^{4+}$, the octahedron is shortened along one axis by about $4 \%$ to accomodate a $\mathrm{d}^{1}$ cation. The system becomes a Mott insulator for $\mathrm{U}=4.8 \mathrm{eV}$ and $\mathrm{J}=0.7 \mathrm{eV}$. The electronic structure is presented in Fig. 1 for $\mathrm{U}=3.5$ $\mathrm{eV}$, where the system is still half-metallic.

Figure 1 shows that half-metallicity occurs with the Fermi level falling within a $2 \mathrm{eV}$ wide band of $\mathrm{Cu} \mathrm{e}_{g}$ and $\mathrm{Vt}_{2 g}$ character, even when degeneracy is broken by the Jahn-Teller distortion. The bottom of the band (a two band complex) is strongly $\mathrm{Cu} \mathrm{d}$ in character, while the $\mathrm{V}$ d character dominates the upper part of the bands.

We expect a small influence of SOC because only $3 \mathrm{~d}$ elements are involved. The full moment compensation that occurs within LSDA+U will be kept when including SOC. This $\mathrm{d}^{9}-\mathrm{d}^{1}$ configuration has small magnetic coupling according to GKA rules (AF according to our calculations), and is spincompensated according to our results. The orbital angular momentum of the $\mathrm{Cu}$ atoms is $0.1 \mu_{B}$, but is negligible for $\mathrm{V}$ and the electronic structure is not affected by the introduction of SOC. Spincompensation remains unaltered when SOC is introduced, thus the total moment of the system is approximately $0.1 \mu_{B} /$ f.u., quite close to the desired result.

\section{B. $\mathrm{Sr}_{2} \mathrm{NiOsO}_{6}: \mathrm{d}^{8}-\mathrm{d}^{2}$}

This material has been synthesized 30 recently and also has been studied using ab initio methods $\underline{31}$ Experimental results were interpreted in terms of a competition between $\mathrm{FM}$ and $\mathrm{AF}$ interactions, caused by the $\mathrm{d}^{8}-\mathrm{d}^{2}\left(\mathrm{Ni}^{2+}-\mathrm{Os}^{6+}\right)$ electronic structure. The closed subshell $\left(\mathrm{d}^{8}\right)$, open subshell $\left(\mathrm{d}^{2}\right)$ should favor half metallicity. According to GKA rules, the $\mathrm{e}_{g}^{2}$-O- $\mathrm{e}_{g}^{0} \mathrm{FM}$ superexchange should be the strongest in this system. However, the magnetic susceptibility data shows both a positive $\Theta_{C W}$ (showing that FM is dominant at high temperature) and an $\mathrm{AF}$ downturn below $50 \mathrm{~K}$, indicating that $\mathrm{AF}$ correlations exist at low temperature. Our ab initio calculations show the FM and AF configurations are similar in total energy, but FM is distinctly the ground state by $20 \mathrm{meV} /$ metal atom (using the experimental structure). Also, the spin-compensation that results within LSDA+U is degraded seriously

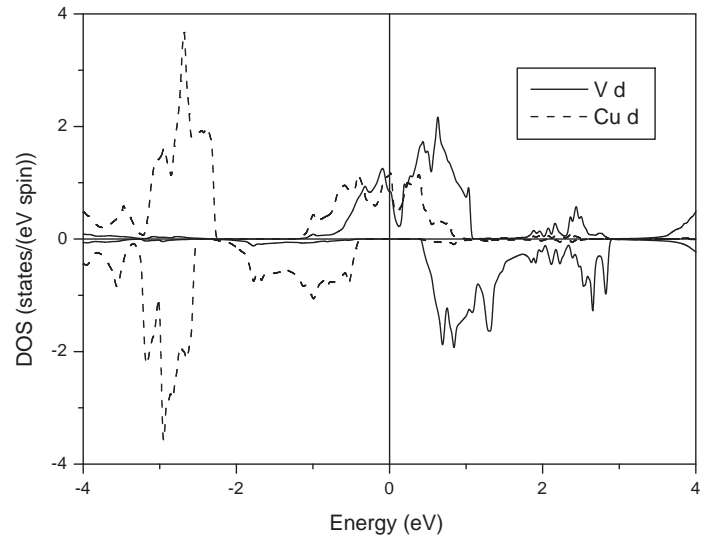

FIG. 1: Density of states plots of the tetragonal double perovskite compound $\mathrm{La}_{2} \mathrm{VCuO}_{6}$ in a $\mathrm{HMAF}$ state within an LSDA+U scheme with $\mathrm{U}=3.5 \mathrm{eV}$ for both $\mathrm{V}$ and $\mathrm{Cu}$. Upper (lower) panel shows up (down) spin channel. The spin moment is precisely compensated.

by SOC, with the large orbital angular momentum leading to a total moment of approximately 1 $\mu_{B} /$ f.u. This value arises from orbital moments of $0.5 \mu_{B}$ for Os and $0.2 \mu_{B}$ for $\mathrm{Ni}$, plus the $0.2 \mu_{B}$ from the total spin moment of the system arising from SOC-induced spin mixing. Hence, the condition of a small magnetic moment is not obtained for this compound. The $\mathrm{d}^{2}-\mathrm{d}^{8}$ electronic structure has the drawback that, when an antialigned spin configuration is set, the orbital angular momenta align and add up rather than (partially) canceling (Hund's third rule for the atoms).

We have performed a structural analysis similar to the one described for $\mathrm{La}_{2} \mathrm{VCuO}_{6}$ with the same preconditions. Comparing with experimental structural data $\stackrel{30}{=}$ our calculated volume is quite good (1\% larger than experiment) and the $c / a$ ratio is $1 \%$ smaller than the experimental value.

TABLE I: Calculated versus experimental structural parameters and selected bond lengths for $\mathrm{Sr}_{2} \mathrm{NiOsO}_{6}$ showing agreement to within $1 \%$ accuracy in the lattice constants.

\begin{tabular}{|c|c|c|}
\hline Parameter & Experimental $^{30}$ & This work \\
\hline \hline$a$ & 5.49 & 5.53 \\
$\mathrm{c}$ & 7.99 & 7.94 \\
Ni-O distance $(\times 4)$ & 2.02 & 1.99 \\
Ni-O distance $(\times 2)$ & 2.04 & 2.03 \\
Os-O distance $(\times 4)$ & 1.91 & 1.92 \\
Os-O distance $(\times 2)$ & 1.96 & 1.94 \\
\hline
\end{tabular}




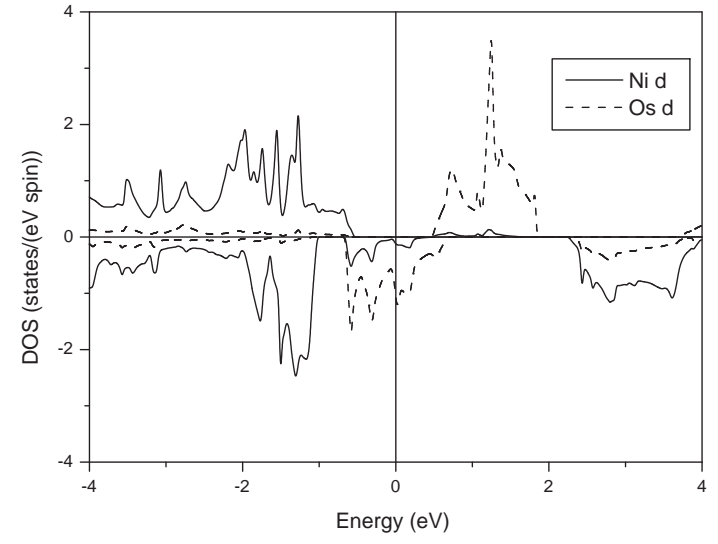

FIG. 2: Density of states plots of the tetragonal double perovskite $\mathrm{Sr}_{2} \mathrm{NiOsO}_{6}$ in a HMAF state within an $\mathrm{LSDA}+\mathrm{U}$ scheme with $\mathrm{U}=5 \mathrm{eV}$ for $\mathrm{Ni}$ and $3 \mathrm{eV}$ for Os, including spin-orbit coupling. Upper (lower) panel shows up (down) spin channel.

For large values of $\mathrm{U}$ this compound becomes a Mott insulator. Half-metallicity occurs only for $\mathrm{U}$ smaller than $3.5 \mathrm{eV}$ for $\mathrm{Os}$ (with $\mathrm{J}=0.7 \mathrm{eV}$ ) and values of $\mathrm{U}$ not larger than $7 \mathrm{eV}$ for Ni. Realistic values of $\mathrm{U}$ and $\mathrm{J}$ would be close to the metal-insulator transition. No experimental information on its conduction properties is available so far. For smaller values of $\mathrm{U}$, we obtain conduction coming from the Os majority spin channel (the unfilled subshell), as can be seen in Fig. 2.

\section{NEW FINDINGS}

Two materials with the desired properties have been clearly identified. Both possess a $\mathrm{d}^{3}-\mathrm{d}^{3}$ electronic configuration so a high-spin, magnetic band insulator situation is a possibility. This particular electronic configuration has several advantages, that we will discuss, over other possible electronic configurations that will promote a spin-compensated state, and they have AF coupling by the GKA rules, so they become plausible candidates for being spincompensated half metals or half semimetals. In addition, we have analyzed a compound with a $\mathrm{d}^{2}-\mathrm{d}^{2}$ configuration, and we discuss distinctions.

\section{A. $\mathrm{Sr}_{2} \mathrm{VReO}_{6}: \mathrm{d}^{2}-\mathrm{d}^{2}$}

This compound is calculated to have a $\mathrm{d}^{2}-\mathrm{d}^{2}$ electronic configuration. The unoccupied $\mathrm{t}_{2 g}$ shells lead to a distortion from cubic symmetry. Relaxing the structure within tetragonal symmetry, the resulting $c / a$ ratio is 1.02 , together with a sizable elongation of the oxygen cages to accommodate the $\mathrm{d}^{2}$ electronic state $(3 \%$ difference in the $\mathrm{V}-\mathrm{O}$ distance and a smaller $1 \%$ difference for the Re-O distance). A summary of the structural parameters obtained is given in Table

TABLE II: Calculated structural parameters for $\mathrm{Sr}_{2} \mathrm{VReO}_{6}$ in the space group I4/mmm (no. 139). $a=$ $5.52 \AA(\sqrt{2} \times 3.90 \AA), c=7.96 \AA(2 \times 3.98 \AA)$.

\begin{tabular}{|c|c|c|}
\hline Atom & Wyckoff position & Atomic parameter \\
\hline \hline $\mathrm{Sr}$ & $4 \mathrm{~d}$ & $(0.5,0,0.25)$ \\
$\mathrm{V}$ & $2 \mathrm{a}$ & $(0,0,0)$ \\
$\mathrm{Re}$ & $2 \mathrm{~b}$ & $(0,0,0.5)$ \\
$\mathrm{O} 1$ & $8 \mathrm{~h}$ & $(0.2521,0.2521,0.5)$ \\
$\mathrm{O} 2$ & $4 \mathrm{e}$ & $(0,0,0.2494)$ \\
\hline
\end{tabular}

We have studied the electronic structure of this yet-unreported compound with both LSDA+U and SOC. Due to slight band overlap, there is substantial but not total spin-moment compensation, leading to a total spin moment of less than $0.1 \mu_{B}$. However, because of the unfilled $t_{2 g}$ shells in both cations, large orbital angular moments develop, particularly large for $\operatorname{Re}\left(0.4 \mu_{B}\right)$. The result is a total magnetic moment of approximately $0.5 \mu_{B}$ for $\mathrm{U}(\mathrm{V})=7$ $\mathrm{eV}$ and $\mathrm{U}(\mathrm{Re})=3 \mathrm{eV}(\mathrm{J}=0.7 \mathrm{eV}$ for both cations). While there is no true gap in the $\mathrm{V}$ majority spin channel, there is a very large fractional spin polarization in the density of states at the Fermi level, as can be seen in Fig. 3 .

\section{B. $\mathrm{K}_{2} \mathrm{MnRhO}_{6}: \mathbf{d}^{3}-\mathbf{d}^{3}$}

As we have just seen in the case of $\mathrm{Sr}_{2} \mathrm{VReO}_{6}$, having unfilled shells encourages orbital angular moments. In that respect, the following two examples are materials which have a $\mathrm{d}^{3}-\mathrm{d}^{3}$ electronic configuration, so the filled $t_{2 g}$ shells will give very small orbital moments to obscure the spin compensation.

We calculate that this $\mathrm{K}_{2} \mathrm{MnRhO}_{6}$ compound has the configuration $\mathrm{Mn}^{4+}: \mathrm{d}^{3}-\mathrm{Rh}^{6+}: \mathrm{d}^{3}$, which couples $\mathrm{AF}$. The $\mathrm{Rh}$ moment is strongly delocalized on the oxygen neighbors (up to $0.3 \mu_{B}$ per oxygen and only $1 \mu_{B}$ in the Rh muffin-tin sphere), but all in all, this compound is near HMAF with Fermi level lying very near band edges. The lattice parameter $a$ was optimized and atomic positions were relaxed within the $\mathrm{Fm} \overline{3} \mathrm{~m}$ space group (no. 225), and $a=$ $3.94 \AA$ was obtained. The $\mathrm{d}^{3}-\mathrm{d}^{3}$ configuration of ions with cubic symmetry leaves a cubic structure, 


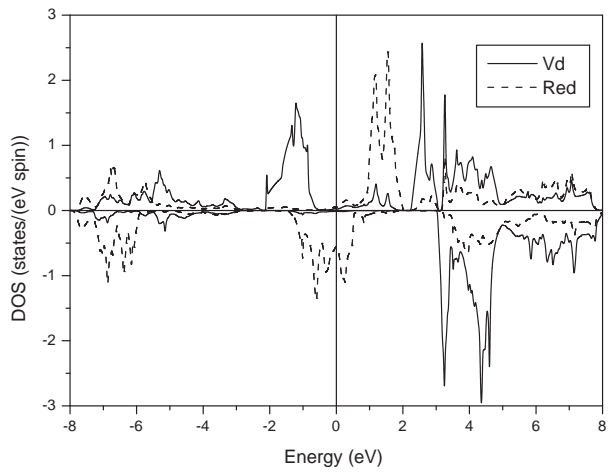

FIG. 3: Density of states plots of the tetragonal double perovskite $\mathrm{Sr}_{2} \mathrm{VReO}_{6}$ in a HMAF state within an LSDA+U scheme with $\mathrm{U}=5 \mathrm{eV}$ for $\mathrm{V}$ and $3 \mathrm{eV}$ for Re, including spin-orbit coupling. Upper (lower) panel shows up (down) spin channel.

leaving only a small relaxation of the oxygen cages around the cations ( $\mathrm{Rh}-\mathrm{O}$ distance of $2.00 \AA$ and Mn-O distance of $1.94 \AA$ ).

As mentioned, very small values of the orbital angular momentum arise when SOC is introduced: $0.05 \mu_{B}$ on Rh, $0.01 \mu_{B}$ on Mn. Adding spin and orbital angular momenta, the net moment is only 0.02 $\mu_{B}$, and it remains very close to compensated half metal for values of $\mathrm{U}$ of $6 \mathrm{eV}$ for $\mathrm{Mn}$ and $4 \mathrm{eV}$ for $\mathrm{Rh}$. Even though Fig. 4 looks similar to a zero-gap situation, on the verge of a metal-insulator transition, bands cross the Fermi level even for larger values of $\mathrm{U}$, and (half) semimetal character is maintained.

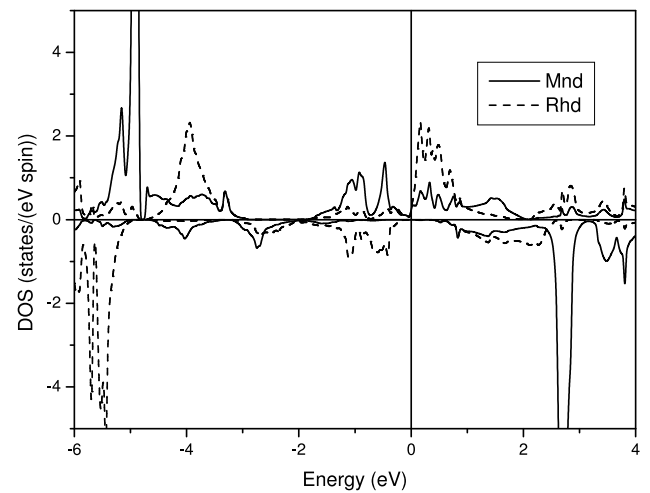

FIG. 4: Density of states plots of the tetragonal double perovskite $\mathrm{K}_{2} \mathrm{MnRhO}_{6}$ in a HMAF state within an $\mathrm{LSDA}+\mathrm{U}$ scheme with $\mathrm{U}=5 \mathrm{eV}$ for $\mathrm{Mn}$ and $3 \mathrm{eV}$ for $\mathrm{Rh}$, including spin-orbit coupling. Upper (lower) panel shows up (down) spin channel.

\section{C. $\mathrm{La}_{2} \mathrm{CrWO}_{6}: \mathrm{d}^{3}-\mathrm{d}^{3}$}

This is another example of a $\mathrm{d}^{3}-\mathrm{d}^{3} \mathrm{AF}$ coupling situation, and again, it is a not-yet-synthesized material, completely ab initio designed. Structural relaxation was carried out allowing tetragonal distortion. No tetragonal distortion was obtained, and $a$ $=4.00 \AA$. The difference in ionic radii leads to a Cr-O distance of $1.97 \AA$ and $\mathrm{W}-\mathrm{O}$ distance of 2.03 $\AA$.

Evolution with U and SOC indicates that the latter does not seriously degrade the moment compensation. For large values of $\mathrm{U}$, where the material is still a metal, the total moment stays below 0.2 $\mu_{B}$ /f.u. W has an orbital moment of $0.15 \mu_{B}$ and a much smaller one, and opposite in an AF configuration, arises from Cr. Even for large values of $\mathrm{U}(7 \mathrm{eV}$ for $\mathrm{Cr}$ and $4 \mathrm{eV}$ for $\mathrm{W}$ ), the system remains metallic (half-metallic) and the total magnetic moment, including spin and orbital components, does not exceed $0.10 \mu_{B}$ /f.u. Metallicity comes from a broad W $\mathrm{d}$ band that crosses the Fermi level, as can be seen in Fig. 5. An electron pocket is formed in an itinerant $\mathrm{W} d$ band and a corresponding La-character hole pocket (not shown) appears.

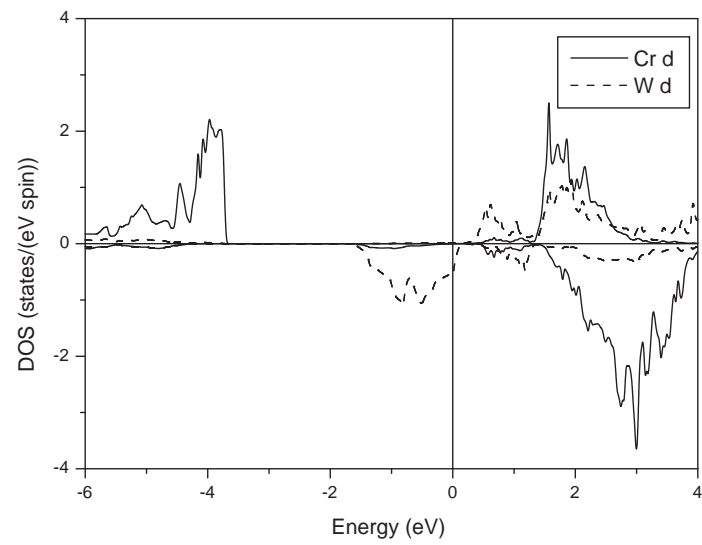

FIG. 5: Density of states plots of the tetragonal double perovskite $\mathrm{La}_{2} \mathrm{CrWO}_{6}$ in a HMAF state within an $\mathrm{LDA}+\mathrm{U}$ scheme with $\mathrm{U}=5 \mathrm{eV}$ for $\mathrm{Cr}$ and $3.5 \mathrm{eV}$ for $\mathrm{W}$, including spin-orbit coupling. Upper (lower) panel shows up (down) spin channel.

\section{UNPROMISING COMPOUNDS}

On our way to detecting these spin-compensated half-metals, we studied other seemingly promising double perovskites. We will discuss these only 
briefly, since they do not show the desired behavior.

\section{A. $\mathbf{d}^{1}-\mathbf{d}^{9}$}

Even though superexchange rules predict this coupling to be negligible, we have previously studied a system that could be promising. Hence, as an analogy with the Jahn-Teller compound $\mathrm{La}_{2} \mathrm{VCuO}_{6}$, we tried changing each of the $3 \mathrm{~d}$ elements by a corresponding $4 \mathrm{~d}$ element, without changing the electron count, i.e. $\mathrm{La}_{2} \mathrm{CuNbO}_{6}$ and $\mathrm{La}_{2} \mathrm{VAgO}_{6}$ which are less likely to be Mott insulators. In these cases, $\mathrm{Nb}$ and $\mathrm{Ag}$ respectively are non-magnetic, and the spincompensation is lost.

\section{B. $\mathbf{d}^{2}-\mathrm{d}^{4}$}

This electronic configuration could lead to a spincompensated state, if the $\mathrm{d}^{4}$ cation is in a low-spin state. We have tried $\mathrm{Ba}_{2} \mathrm{FeCrO}_{6}$ (also with $\mathrm{Sr}$ and Ca with similar results), which could assume a $\mathrm{d}^{2}-\mathrm{d}^{4}$ electronic configuration. It is indeed the case, but Fe is in a HS state (not allowing for spin compensation to happen). A similar problem appears with the compound $\mathrm{K}_{2} \mathrm{FeRuO}_{6}$, where also $\mathrm{Fe}^{4+}$ is also in a HS state. This electronic configuration has been studied in the past ${ }^{11,12}$ for several perovskites with general formula $\mathrm{AA}^{\prime} \mathrm{BB}^{\prime} \mathrm{O}_{6}$.

\section{C. $\mathbf{d}^{2}-\mathrm{d}^{8}$}

We have found several cases where always the FM exchange mediated by the $\mathrm{e}_{g}$ electrons is more intense than the AF coupling between the $\mathrm{t}_{2 g}$ electrons. Starting from $\mathrm{Sr}_{2} \mathrm{NiOsO}_{6}$ compound discussed above and substituting $\mathrm{Os}$ by $\mathrm{Ru}$ to form $\mathrm{Sr}_{2} \mathrm{NiRuO}_{6}$, we find that FM coupling remains. This is the standard prediction of GKA rules. Apart from these, we have also identified that FM coupling is more favorable in $\mathrm{La}_{2} \mathrm{CrNiO}_{6}$ (and also with $\mathrm{W}$ and Mo on the $\mathrm{Cr}$ site). This material is calculated to be an interesting FM insulator (of which there are relatively few examples), where instead of promoting the possible Jahn-Teller distorted $\mathrm{Ni}^{3+}$ cation, it leads to the formation of a $\mathrm{Cr}^{2+}-\mathrm{Ni}^{4+}$ configuration. This charge ordered state is thought to occur to prevent the system from developing a Jahn-Teller distortion when it is close to the itinerant electron limit 32

Disordered $\mathrm{LaCr}_{0.5} \mathrm{Ni}_{0.5} \mathrm{O}_{3}$ is known experimentally to be insulating, 33 but close to a metalinsulator transition (the $\mathrm{Ni}$ end of the series $\mathrm{LaNiO}_{3}$ is a metal). Our calculations show that the electronic structure of the charge ordered material explains the insulating state found experimentally, together with the prediction of FM coupling between the $\mathrm{d}^{2}$ and $\mathrm{d}^{8}$ cations, making the system potentially interesting as another FM insulator. For these calculations, we have taken the lattice parameters from the experiment ${ }^{34}$ with the disordered system and relaxed only the internal coordinates. The different ionic radii lead to different metal-oxygen bond lengths (1.90 $\AA$ for $\mathrm{Cr}$ and $1.96 \AA$ for $\mathrm{Ni}$ ).

\section{Antiferrimagnetic insulators}

We have found the following magneticallycompensated insulators with double perovskite structure: i) $\mathrm{La}_{2} \mathrm{CrMoO}_{6}$ is isoelectronic to the spin-compensated metal $\mathrm{La}_{2} \mathrm{CrWO}_{6}$, but introducing Mo instead of $\mathrm{W}$ opens up a gap at the Fermi level. It is the greater itineracy of the $5 \mathrm{~d}$ element that leads to the metallic state that we analyzed above. ii) In analogy with $\mathrm{Sr}_{2} \mathrm{CrOsO}_{6}, \stackrel{8}{=}$ we have tried with a bigger alkaline-earth cation. The compound $\mathrm{Ba}_{2} \mathrm{CrOsO}_{6}$ is found to be an antiferromagnetic insulator, with configuration $\mathrm{d}^{3}-\mathrm{d}^{3}$. As an isovalent compound, $\mathrm{Sr}_{2} \mathrm{CrRuO}_{6}$ is calculated to be an antiferromagnetic insulator.

\section{SUMMARY}

In this paper we have suggested two very nearly spin-compensated half metals (or half semimetals): $\mathrm{K}_{2} \mathrm{MnRhO}_{6}$ and $\mathrm{La}_{2} \mathrm{CrWO}_{6}$, both with a formally $\mathrm{d}^{3}-\mathrm{d}^{3}$ configuration. It is found that, within a double perovskite structure, a $\mathrm{d}^{3}-\mathrm{d}^{3}$ electronic configuration promotes the chances to obtain a nearly spincompensated half-metal, due to several factors. i) No competition between FM and AF interactions occurs as happens when there is partial occupation of $\mathrm{e}_{g}$ states in one of the metal cations. ii) Having the $t_{2 g}$ shell completely filled leads to a very small orbital angular moment, which works even further in favor of a very small magnetic moment solution. iii) Having less than half-filled d shells in both metal ions helps to avoid imbalance in the total moment that occurs when spin-orbit coupling is important, specially for $4 \mathrm{~d}$ and $5 \mathrm{~d}$ elements. Having both cation d shells less than half filled means that the orbital angular momenta of both cations will also orient antiparallel to the spin, preventing the system from having a larger net magnetic moment. iv) The large $\mathrm{S}=3 / 2$ spins make our calculations more realistic; ordering of low spin ions is often more affected by quantum fluctuations. Both materials that we have 
discussed at length are designed $a b$ initio, no experimental information on them yet exists to the best of our knowledge.

\section{ACKNOWLEDGMENTS}

We have benefited from discussion on this topic with J. Chakhalian. This project was supported by DOE grant DE-FG02-04ER46111 and through interactions with the DOE's Predictive Capability for Strongly Correlated Systems team of the Computational Materials Science Network. V.P. acknowledges financial support from Xunta de Galicia through the Human Resources Program.

* Electronic address: victor.pardo@usc.es

${ }^{1}$ K. I. Kobayashi, T. Kimura, H. Sawada, K. Terakura, and Y. Tokura, Nature 395, 677 (1998).

2 M. I. Katsnelson, V. Yu. Irkhin, L. Chioncel, A. I. Lichtenstein, and R. A. de Groot, Rev. Mod. Phys. 80, 315 (2008).

3 J. Chakhalian, J. W. Freeland, G. Srajer, J. Strempfer, G. Khaliullin, J. C. Cezar, T. Charlton, R. Dalgliesh, C. Bernhard, G. Cristiani, et al., Nat. Phys. 395, 677 (1998).

4 H. van Leuken and R. A. de Groot, Phys. Rev. Lett. 74, 1171 (1995).

${ }^{5}$ W. E. Pickett, Phys. Rev. Lett. 77, 3185 (1996).

${ }^{6}$ W. E. Pickett and H. Eschrig, J. Phys.: Condens. Matt. 19, 315203 (2007).

7 K. W. Lee and W. E. Pickett, Europhys. Lett. 80, 37008 (2007).

${ }^{8}$ K. W. Lee and W. E. Pickett, Phys. Rev. B 77, 115101 (2008).

${ }^{9}$ G. Jackeli and G. Khaliullin, Phys. Rev. Lett. 102, 017205 (2009).

10 W. E. Pickett, Phys. Rev. B 57, 10613 (1998).

11 J. H. Park, S. K. Kwon, and B. I. Min, Phys. Rev. B 65, 174401 (2002).

12 Y. K. Wang and G. Y. Guo, Phys. Rev. B 73, 064424 (2006).

13 M. S. Park and B. I. Min, Phys. Rev. B 71, 052405 (2005).

14 M. Nakao, Phys. Rev. B 74, 172404 (2006).

15 I. Galanakis, K. Ozdogan, E. Sasioglu, and B. Aktas, Phys. Rev. B 75, 172405 (2007).

16 I. Galanakis, P. H. Dederichs, and N. Papanikolaou, Phys. Rev. B 66, 134428 (2002).

17 I. Galanakis, P. H. Dederichs, and N. Papanikolaou, Phys. Rev. B 66, 174429 (2002).

18 P. C. Canfield, Nature Physics 4, 167 (2008).

19 V. I. Anisimov, J. Zaanen, and O. K. Andersen, Phys. Rev. B 44, 943 (1991).

20 E. R. Ylvisaker, W. E. Pickett, and K. Koepernik, Phys. Rev. B 79, 035103 (2009).

21 J. B. Goodenough, Magnetism and the chemical bond (IEEE Press, New York, 2001).

22 P. Hohenberg and W. Kohn, Phys. Rev. 136, B864 (1964).

23 K. Schwarz and P. Blaha, Comp. Mat. Sci. 28, 259 (2003).

24 E. Sjöstedt, L. Nördstrom, and D. J. Singh, Solid State Commun. 114, 15 (2000).

25 J. P. Perdew and Y. Wang, Phys. Rev. B 45, 13244 (1992).

26 A. I. Liechtenstein, V. I. Anisimov, and J. Zaanen, Phys. Rev. B 52, R5467 (1995).

27 E. R. Ylvisaker, W. E. Pickett, and K. Koepernik, Phys. Rev. B 79, 035103 (2009).

28 J. P. Perdew, K. Burke, and M. Ernzerhof, Phys. Rev. Lett. 77, 3865 (1996).

29 R. E. Rudd and W. E. Pickett, Phys. Rev. B 57, 557 (1998).

30 R. Macquart, S. J. Kim, W. R. Gemmill, J. K. Stalick, Y. Lee, T. Vogt, and H. S. C. zur Loye, Inorg. Chem. 44, 9676 (2005).

31 W. Song, E. Zhao, J. Meng, and Z. Wu, J. Chem. Phys. 130, 114707 (2009).

32 I. I. Mazin, D. I. Khomskii, R. Lengsdorf, J. A. Alonso, W. G. Marshall, R. M. Ibberson, A. Podlesnyak, M. J. Martinez-Lope, and M. M. AbdElmeguid, Phys. Rev. Lett. 98, 176406 (2007).

33 P. Ganguly, N. Y. Vasanthacharya, C. N. R. Rao, and P. P. Edwards, J. Solid State Chem. 54, 400 (1984).

${ }^{34}$ G. J. Zhang, R. Liu, Y. Yang, and Y. Q. Yia, phys. status sol.(a) 160, 19 (1997). 